\title{
Foreword to the Thematical Issue "Methods for Design of the Control Systems Based on Dynamic Models"
}

DOI: $10.1134 /$ S0005117911100018

The present thematical issue of "Automation and Remote Control" includes selected papers based of the materials of the session "Methods for Design of the Control Systems Based on Dynamic Models" of the Second Russian Conference with International Participation "Hardware and Software of the Systems of Control, Checking, and Measurement" (UKI-10), Moscow, Trapeznikov Institute of Control Sciences, Russian Academy of Sciences, October 18-20, 2010 (Chairman of the Organizing Committee Ambartsumyan A.A.).

The papers of this issue fall into the three groups:

- methods for construction of the control algorithms;

- methods and practice of constructing the algorithms to control plants of various physical nature (flight vehicles, robots, power systems);

- software for the methods for construction of the control algorithms and industrial controllers to realize them;

Papers of the first group are as follows:

- "Design of the Digital $H_{\infty}$-controllers for Multivariable Systems of Prescribed Precision According to the RMS Criterion."

- "Design of the $H_{\infty}$-controllers of Multivariable Systems of Prescribed Precision and Degree of Stability."

- "Compensation of Perturbations and Noise at Control of a Linear Plant."

- "Moving Control of Oscillations in the Distributed-parameter Systems."

- "Optimization of the Quasilinear Complex Dynamic Stochastic Systems."

The second group includes the following papers:

- "Design of Invariant Control System for Longitudinal Motion of Flight Vehicle."

- "Method of Decomposition in the Problems of Mobile Robot Control."

- "Multidimensional System of Cascaded Control of Plasma Form and Current in Tokamak with Channel Decoupling and $H_{\infty}$-controller."

- "Design of the Discrete Controllers of Reduced Dimensionality for the Distributed Follower."

- "Pole Arrangement at Controlling a Large Power System."

The third group includes the following papers:

- "GAMMA-3 System and its Application."

- "Self-adjusting PID/I-controller."

The compilers of the present issue expect that it might be useful for the researchers and engineers designing the up-to-date automatic control systems.

A.G. Aleksandrov 